\title{
Fractional Order PID Control mechanism for Helicopter Gearbox Test Control with internal and External Disturbance
}

\author{
Somayeh Dolatkhah TAKLOO ${ }^{1, *}$, Shadan MoZAFARI ${ }^{2}$, Mehdi REZAZAdEHMOHAMAdi $^{3}$, \\ and Mohsen MARDANI ${ }^{4}$ \\ ${ }^{1}$ Amirkabir University of Technology - Tehran Polytechnic, Tehran, Iran. \\ Email: somayeh.dolatkhah@ gmail.com \\ ${ }^{1,2,3,4}$ Sharif University of Technology branch of ACECR \\ (Academic Center of Education, Culture and Research)
}

\begin{abstract}
Fractional order PID (FOPID) controller is a special kind of PID controller which it's derivative and integral order are fractional rather than integer and has five parameters. Using this control improves the control results in accuracy and speed. Statistics show that using this controller for systems with high sensitivity is more practical and safe. Mechanical closed loop test rig acts an important role in aviation industry. Fault diagnosis in gearboxes and other critical components of the airplane and helicopters is the most important part of this industry. More over testing rotating equipment is very important for system security verification. There are several testing technics and many important testing parameters. One of these essential factors is gearbox security test technique. What's more, mechanical closed-loop system is a very cost effective method. For financial, energy consumption and maintenance reasons, using a system with the ability of energy regeneration is suggested. In this paper mechanical close loop with energy regeneration mechanism is used for testing the helicopter gearbox. Two kinds of controllers are used for keeping the closed-loop's torque in the constant value. The result of paper shows the behavior of PID and FOPID controller methods in controlling torque circulating in this mechanical closed loop. Beside the overshoot and settling time is considerable.
\end{abstract}

Keywords: Test rig, PID Controller, FOPID Controller, Mechanical Close Loop System.

\section{Introduction}

Due to the importance of gearboxes tasks in aeronautic industry, testing and fault detection are vital and undeniable parts of their safety check list. Closed-loop test rigs are more preferred than open-loop ones because of their lower energy losses. Also among different closed-loop rigs, mechanical ones have lower costs especially than the electrical ones. In the presented setup that is made for testing orthogonal gearboxes, the idea of energy circulation in a closed loop is used. In some of studies open and close loop systems are used for gearbox test. Also in some papers lots of parameters is observed for diagnosis of any kind of fault in gearboxes. Beside in some methods power deviation and close loop optimization is studied. 
Also so many closed loop test rigs have been made and different uses of them have been defined and studied.

The presented test rig is a special kind of noted rigs suitable for testing orthogonal gearboxes in which, hydraulic jack and planetary gearboxes are used for application of locked torque into the system. Torsion is applied to the ring of the planetary gear due to the displacement of pistons in hydraulic cylinders. so a locked torque is applied into the system during rotation of components due to this torsion. The rotational speed is required for producing power which is supplied by an electric motor which is controlled by a drive. Torque and rotational speed can be adjusted due to the determined tests. Precise testing condition is achievable due to possibility of controlling of each parameter. Power is transferred by shafts and gearboxes and the couplings and connections prevent transmission of moment to gearboxes. Because of loop bounding, the energy is circulated inside the loop and the motor is just responsible for developing the maximum energy needed at the first moment of setting up the system and preventing energy wasting along the loop during the rest of the process. The system is also dynamically investigated and designed such that the frequencies and vibrational behavior of the system and the gearboxes are completely separate from each other. So using the present test rig, accurate fault detection and testing of gearboxes in their operational condition can be performed with precise dependent controlling of torque and speed. The results show that the conceptual design of the present closed-loop gearbox test rig can meet design requirements and safety criterion.

One of the most important components of this system and its control section is torque inducer part. In some articles the methods of the torque inducer control in mechanical close-loop are studied. In these systems torque is applied by applying torsion to the loop in different ways. Among these methods, using of hydraulic actuator to provide the necessary force for torque inducer system or an electric drive system with gearbox is more popular. [1, 2, 3, 4] There are many different ways for controlling these kinds of hydraulic actuators. Hydraulic actuators are known for their high power and have an important role in modern machine equipment. However, these behaviors are due to their complex nonlinear dynamics. This non-linear behavior can be caused by friction, compressibility of the fluid in the hydraulic actuator or other reasons. Using these actuators is possible and more comfortable by using modern digital control systems which are designed based on modern control theory. The torque control is possible in different methods including actuator feedback controller design pressure, position control, speed of shaft movement, etc. Electronic proportional actuators and servo valves are used in the design of the controller drivers. Moreover, positional converters and force sensors are used as feedback for making close loop control systems. In an effort to improve the performance of PID tuning for processes with changing dynamic properties, in this study automatic tuning based on Ziegler Nichols tuning method is applied. This algorithm will search for optimal values of $\mathrm{Kp}, \mathrm{Ki}$ and $\mathrm{Kd}$ from a given specified step response requirements and actuator constraints. Besides, in FOPID controller method 5 parameters are tuned by foncom matlab tuning tool. The remainder of this paper is organized as follows: In Section 2, the system mechanical closedloop is proposed and the conventional systems is shown. In Section 3, problem formulation and dynmic model is extracted and Section 4 presents the procedure of designing the force controller. Section 5 shows the simulation and experimental results of the load simulator force control. Conclusions are presented in Section 6. 


\section{Mechanical closed loop system}

In recent years use of mechanical closed loop systems which is shown in figure 1 is increased.

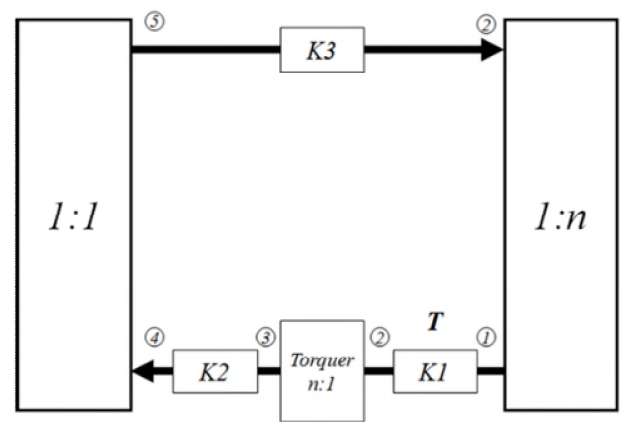

Fig. 1: Mechanical closed loop system with torque induction mechanism

As shown in figure 2 energy circulates inside the mechanical parts of the loop. In mechanical close loop systems a driver is considered for setting up the system which undertakes the task of compensating loss of component. As specified before in the mechanical closed-loop methods energy consumption is much lower than in open looped ones because of energy recovery and also because heat is the only consumed energy loss in different parts of the loop. In contrast, the number of mechanical components which are used in the loop is considerable in comparison with the other methods. [5]

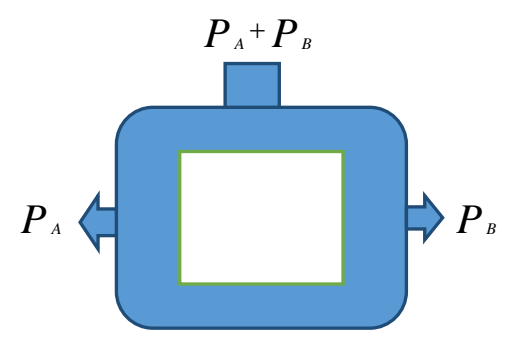

Fig. 2: Mechanical close loop losses

Mechanical closed-loop method or four squares is made of a chain of mechanical components that the beginning and the end of the chain since connect them to each other should have absolutely identical rotational speed. In order to produce circulating power in the closedloop we should providetorque and rotational speed independently in the process. Since all components are engaged with each other in a closed loop for creating torque we should form locked torque proportional to tosionsand torsional stiffnesses of the closed-loop components before all components are connected to each other. For creating the second factor for producing power (rotational speed) we could make motion from the arbitrary point in closed-loop so all the components start moving.

The mechanical close loop is made of three parts:

Power generation

Power transmission

Torque induction 


\section{Power generation section}

If components in loop are used with full efficiency, primary motion and by overcoming to inertia then all the components in the closed-loop could have been tested under the load and without spending energy.

\section{Power transmission}

Mechanical closed loop chain in figure 3 is composed of a series of mechanical components which is used for power transmission tasks and they also could have been tested in the chain. [5] If we could test several similar pieces at the same time we should consider that the amount of power which is received by the components is reducing in the power flow direction. Locked torque is essential in closed loop systems. Therefore, we should produce this amount of torque in close loop systems.

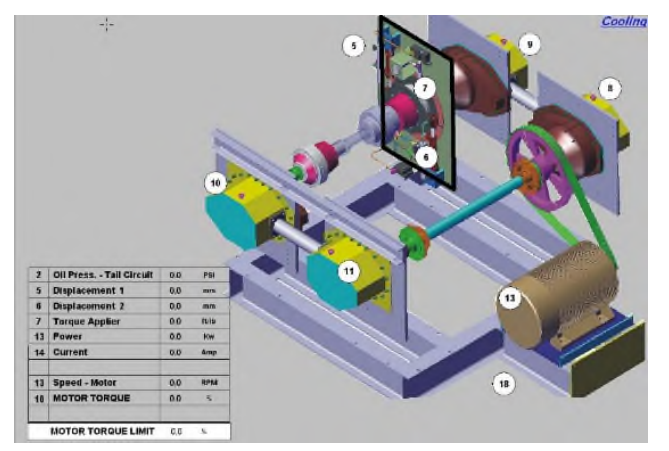

Fig. 3: Mechanical Closed Loop Test Rig

As discussed, torsion in mechanical close loop for producing locked torque is necessary. The required mechanism by which locked torque is generated in mechanical closed loop is called torque induction system as shown in figure 4 .

There are two methods for inducing torque as below:

Static

In static method for making specific torque the close loop should be in static position. Therefore, torque changing during process is impossible. The most simple torque induction mechanism is shown in figure 5 which is made of two opposing disk with wholes on their outsides.

The other disk is twisted for making specified torque induction. Two disks are connected to each other by wholes on their body.

In dynamic methods, static position for close loop system is not required. The induction torque method in dynamic mechanism is made in different ways: helical gearbox, hydraulic mechanism, differential mechanism and planetary method.

As we mentioned before the planetary method is used in this article for torque induction operation. In this method as shown in figure 3 planetary gearbox made of sun and planetary gearboxes and rings. In typical applications the planetary rings attached to its crust. If the gear ring spins around gearbox axis gearbox input and output axis rotates in their opposite side and cause angle difference proportional to their ratio. This method is the most common way to create torque in closed loop. 


\section{Problem formulation and dynamic models}

The equivalent stiffness and rotational inertia are required for achieving the system's transfer function. The system model in AMESIM software is shown in figure 4. In this modeling the spring is used instead of each transmission component. The equal stiffness can be reached by equation 1 :

$$
\frac{1}{k_{e q}}=\frac{1}{k_{1}}+n^{2}\left(\frac{1}{k_{2}}+N^{2}\left(\frac{1}{k_{3}}+\frac{1}{N^{2}}\left(\frac{1}{k_{4}}+\frac{1}{k_{5}}+N^{2}\left(\frac{1}{k_{6}}+\frac{1}{N^{2}}\left(\frac{1}{k_{7}}\right)\right)\right)\right)\right)
$$

Where $\mathrm{k}_{\mathrm{i}}$ refers to different torsional stiffnesses.

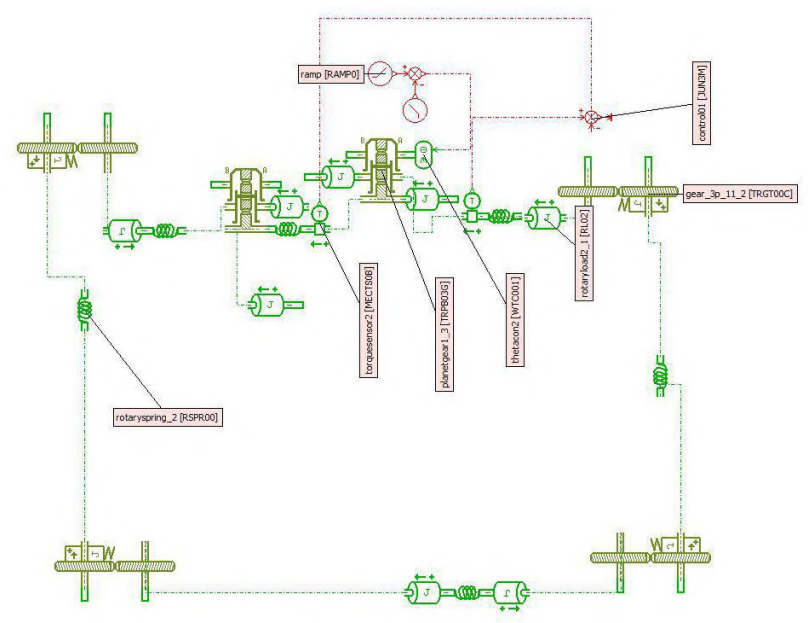

Fig. 4: Schematic model of mechanical closed-loop test rig

The equal inertia could be derived from equation 2 :

$$
\begin{aligned}
& J_{e q}=2 j_{s}+J_{1}+\left(\frac{1}{n}\right)^{2}\left(J_{p}+J_{g 1}+J_{2}+\left(\frac{1}{N}\right)^{2}\left(J_{g 2}+J_{2}+J_{g 2}+(N)^{2}\left(J_{g 1}+J_{4}+J_{g 1}+\right.\right.\right. \\
& \left.\left.\left.\left(\frac{1}{N}\right)^{2}\left(J_{g 2}+J_{2}+J_{g 2}+(N)^{2}\left(J_{g 1}+J_{2}+J_{p c}\right)\right)\right)\right)\right)
\end{aligned}
$$

Where $\mathrm{J}_{\mathrm{i}} \mathrm{s}$ refer to inertia moment of each gear. As mentioned Torque control is our finale goal. Motor speed changes will cause some distraction in close loop. This distraction is not desired for our system and we infer it as a fault in our system which should be disappeared. This distraction is made by aviation in speed of drive motor. Therefore, we should eliminate this distraction by rotating the planetary gear's ring. For controlling the value of the torque in the mechanical closedloop systems we need system transfer function for controlling it with the proper controller. Our transfer function relies on valve, controller, hydraulic jacks and planetary gearbox model. In torque induction system two hydraulic actuators as shown in figure 5 are used for rotating the planetary gear ring. Motor cycles are equal to first shaft cycle. So, first should derive the relations between points' rotations and basis point's rotation. 


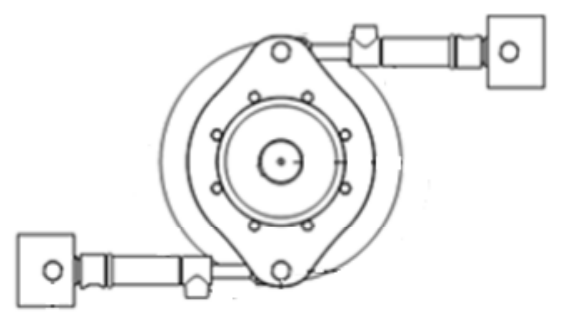

Fig. 5: Hydraulic actuator

According to proportional ratio of gearboxes between these two points we have:

$\theta_{m}=\theta_{1}=\theta_{3}$

$\theta_{4}=3.4 \theta_{3}$

$\ddot{\theta}_{4}=3.4 \ddot{\theta}_{m}$

Where $\ddot{\theta}_{m}$ basis point speed is changing rate and $\theta_{m}$ is motor's shaft rotation. In this test at the beginning, torque is considered equal to the set point. Then torque is start changing by motor speed changes and with respect to drive resistant to motor sudden speed changes and keeps it in the specific rate. These changes in torque values should be controlled by controller. Resistance torque with respect to rotational speed change rate is reachable by having the entire system's rotary inertia proportional based on the basis point. This torque according to motor speed change is equal to $j_{e q} \cdot \ddot{\theta}_{m}$. The current control valve transfer function according to MOOG company control valve catalogue [6] is equals to:

$$
\frac{Q(s)}{i}=\frac{k_{s v}}{1+2 \varsigma\left(\frac{s}{\omega_{s}}\right)+\left(\frac{s}{\omega_{s}}\right)^{2}}
$$

Where $k, \omega_{n}, \xi$ are valve static gain, natural frequency and damping factor.

The torque required by torque induction system should be equal to torque generated by the motor speed changes rate. Moreover, maximum stroke length of jacks is very important and should not be exceeded. Induced torque is corresponds with the amount of jacks stroke.

Actually, input is the current control valve flow and output is the induced torque from torque induction system. What's more, the torque caused by motor cycle change rate could be considered as set point. System transfer function which is the proportional between inputs and outputs (flow, torque) could be reached as follows [6]:

$$
V=\int Q d t
$$

$\Delta T_{\text {oropuer }}=2\left(\int i\left(k_{s v} \frac{\omega_{n}}{\sqrt{1-\zeta^{2}}}\right) e^{-\zeta \omega_{n} t} \sin \left(\left(\sqrt{1-\zeta^{2}}\right) \omega_{n} t\right) d t\right)\left((n-1) k_{\text {eq }} / A D\right)=2 i\left(k_{s v} \frac{\omega_{n}}{\sqrt{1-\zeta^{2}}}\right)$

$\left(\frac{e^{-\zeta \omega_{n} t}\left(-\zeta \omega_{n} \sin \left(\left(\sqrt{1-\zeta^{2}}\right) \omega_{n} t\right)-\left(\sqrt{1-\zeta^{2}}\right) \omega_{n} \cos \left(\left(\sqrt{1-\zeta^{2}}\right) \omega_{n} t\right)\right)}{\left(\zeta \omega_{n}\right)^{2}+\left(1-\zeta^{2}\right) \omega_{n}^{2}}(n-1) k_{e q} / A D=\right.$

$\frac{T}{i}=2 k_{s v} \omega_{n} e^{-\zeta \omega_{n} t}\left(\frac{e^{-\zeta \omega_{n} t}\left(-\zeta \omega_{n} \sin \left(\left(\sqrt{1-\zeta^{2}}\right) \omega_{n} t\right)-\left(\sqrt{1-\zeta^{2}}\right) \omega_{n} \cos \left(\left(\sqrt{1-\zeta^{2}}\right) \omega_{n} t\right)\right)}{\left(\zeta \omega_{n}\right)^{2}+\left(1-\zeta^{2}\right) \omega_{n}{ }^{2}}\right)\left((n-1) k_{e q} / A D\right)$

Where $Q_{p}$ is the pump flow rate. 
Hence, we could reach to the system transfer function with proportional valve for using PID controller.

In control input there are some potential noises which makes fault in system controlling. The figure 6 shows structure of PID controller in presents of noise and distraction. Simulation results for input noise and external disturbance are discussed in section 5 .

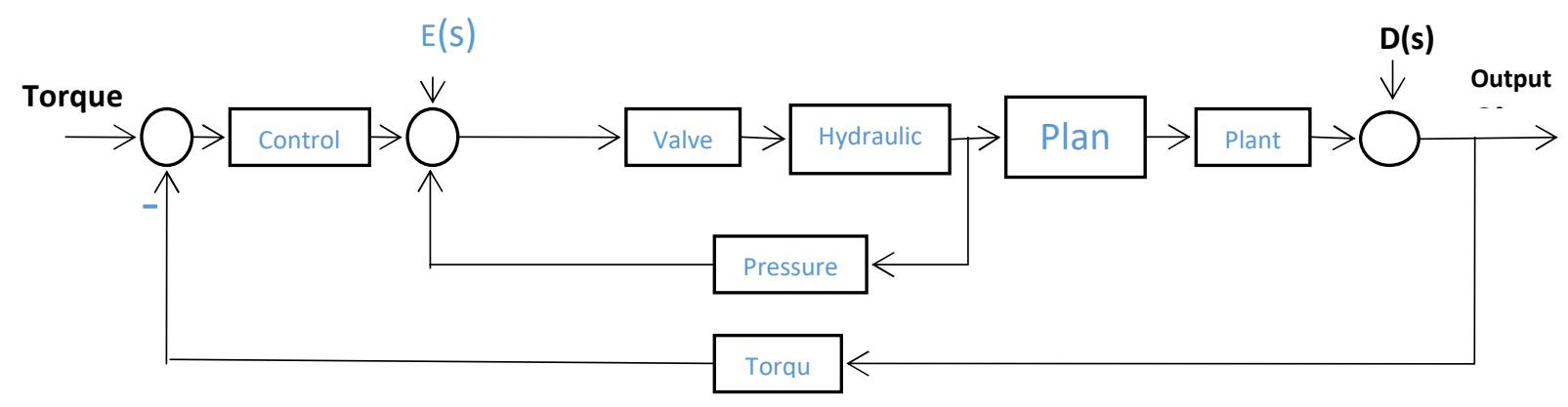

Fig. 6: Torque Control Loop in Presence of Noise and Distraction

\section{Controler design}

\subsection{PID}

PID controller is by far the most common control algorithm. Most practical feedback loops are based on PID control or some minor variations of it. Many controllers do not even use derivative action. The PID algorithm can be approached from many different directions. It can be viewed as a device that can be operated with a few empirical rules, but it can also be approached analytically. PID-controller is the most common controller in many industrial applications and it has been stated in many papers that a PID-controller has been used in hydraulic position servo systems $[8,9,10$, and 11]. This controller type provides proportional with integral and derivative control. This controller combines proportional control with two additional adjustments, which helps the unit automatically compensate for changes in the system.

The used PID controller in this paper is tuned by Ziegler Nichols methods. The proportional, derivative and integral coefficients in this methods are compared with the factors which are catched from the matlab self tunning PID. The results show that the paramters which are reached from Ziegler Nichols are the best ones and this improves the control tracking reults. 


\subsection{FOPID}

The performance of the PID controller can be improved by use of fractional order derivatives and integrals. This flexibility helps to design a more robust system. The most important advantages of the $P I^{\lambda} D^{\mu}$ controller are the better control of dynamical systems and less sensitivity to changes of parameters of a control system [12]. Before using the fractional order controller in the design, an introduction to the fractional calculus is required. PID controller, has been demonstrated by Oustaloup [13]. Podlubny has proposed a generalization of the PID controller as $P I^{\lambda} D^{\mu}$ controller which is known as fractional order PID controller, where $\lambda$ is the non-integer order of integrator and $\mu$ is the non-integer order of the differentiator term. He also demonstrated that the $P I^{\lambda} D^{\mu}$ controller has better response than classical PID controller [14]. Frequency domain approaches of $P I^{\lambda} D^{\mu}$ controller are studied in [15]. Also many valuable studies have been done for fractional order controllers and their implementations in [16].

\section{Simulation results}

To test the feasibility of the developed controller, co-simulation based on AMESim and MATLAB/Simulink was carried out. As discussed, in order to verify model parameters, the load simulator model was built in AMESim and developed controller was achieved in Matlab. In figure 7 the model response into the different inputs for PID and FOPID controller are shown. All PID factors are reached by Ziegler Nichols tuning method for evaluation each procedure. FOPID parameters also are computed in Matlab tuning toolbox. What's more, Figure 7 shows the optimization progress for each iteration and found an optimal solution within the specified tolerances. Steady state error and peak overshoot have been reduced to near to zero, settling time and rise time has also been reduced in FOPID controller technique. As discussed before in both methods the results in presence of the internal noise and external disturbance for different inputs are compared with each other.



a) PID without noise and disturbance Step Set Point 
Bulletin de la Société Royale de Liège, Vol. 86, special issue, 2017, p. 127 - 138

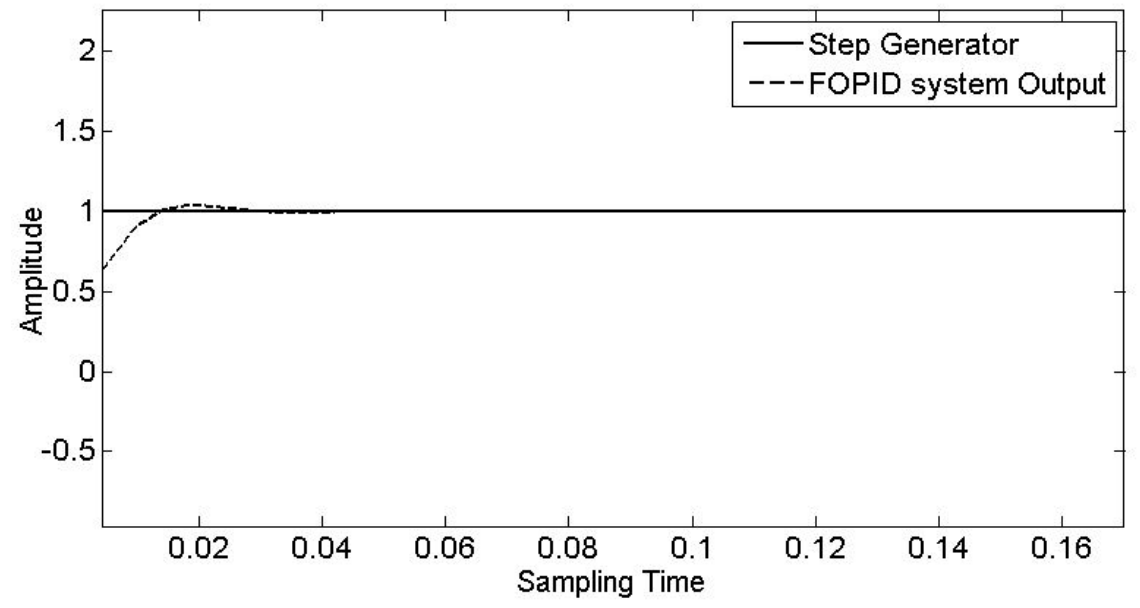

b) FOPID without noise and disturbance Step Set Point



c) PID Controller with internal noise Pulse Set Point

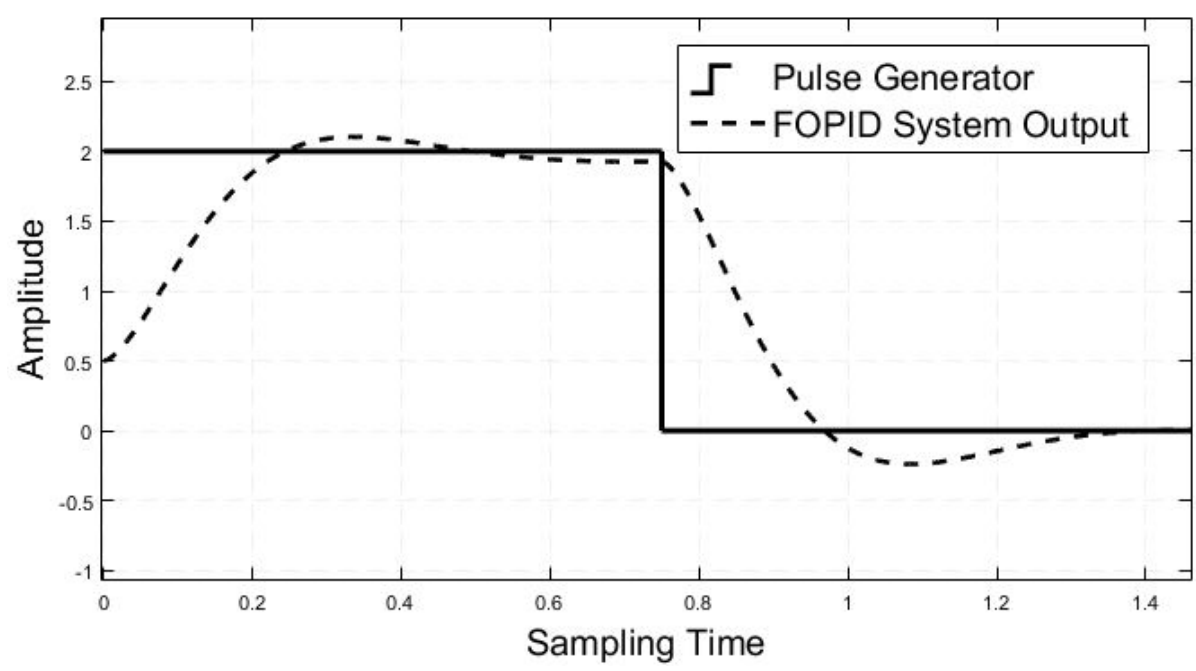

d) FOPID Controller with internal noise Pulse Set Point 


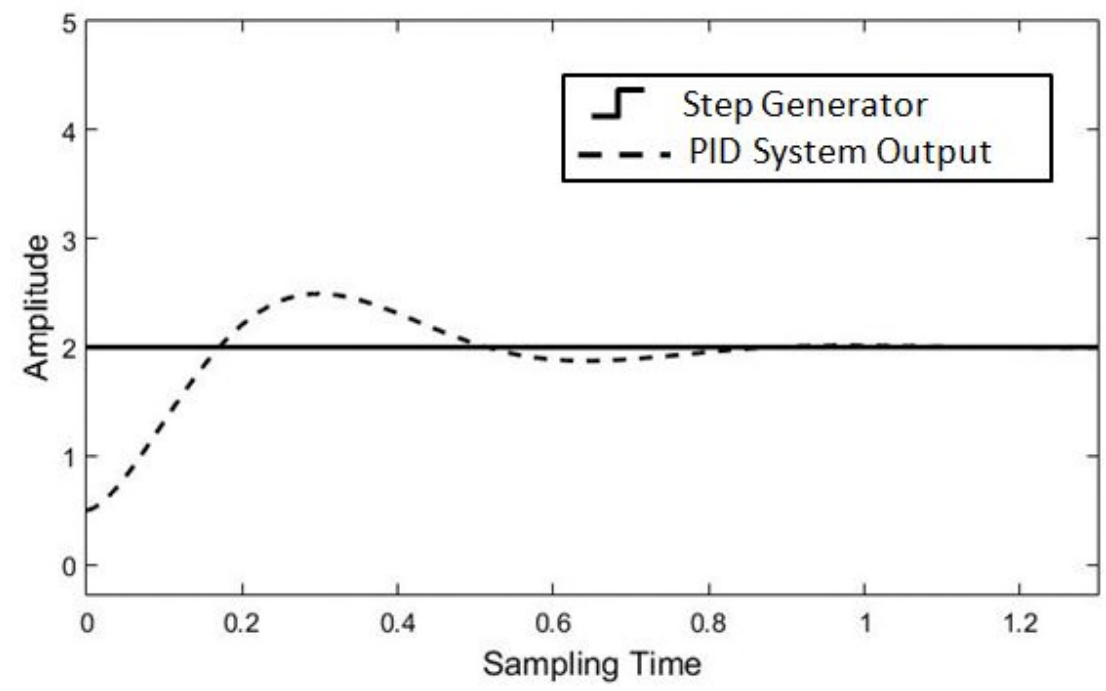

e) PID Controller with internal noise and external disturbance Step Set Point

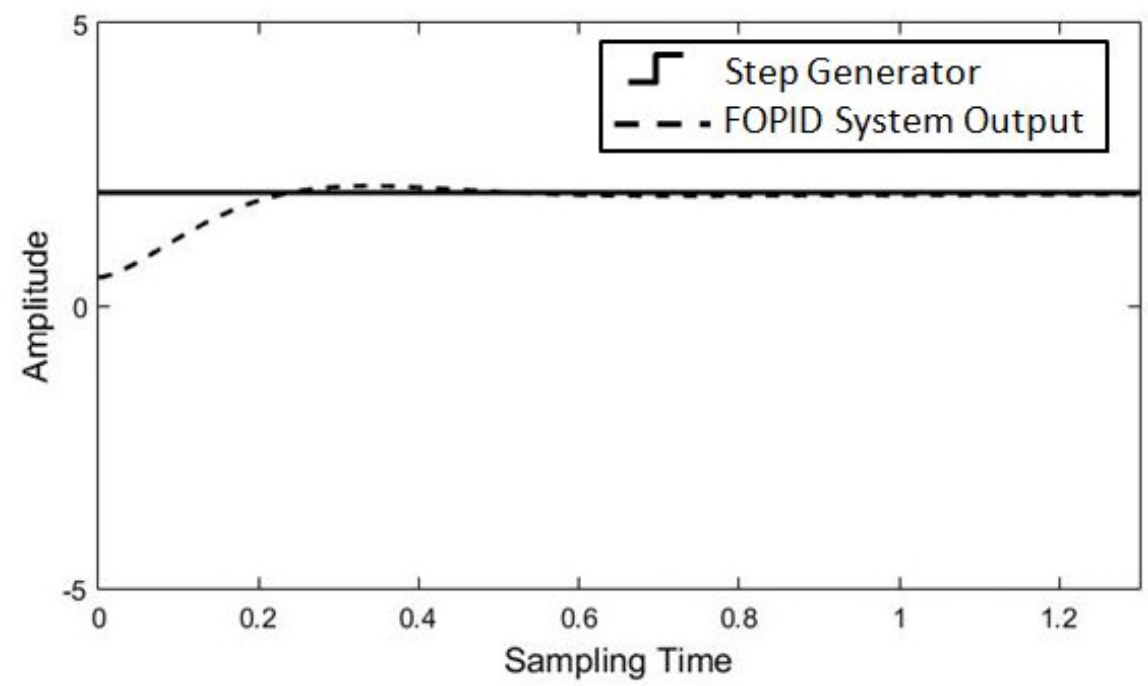

f) FOPID Controller with internal noise and external disturbance Step Set Point Simulation results of PID and FOPID controller in presence and absence of internal noise and external disturbance for different input trajectories

As is shown critical parameters could have been adjusted by the PID factors. Based on the error analysis, control effort and observation on the tracking performance, the PID control provides more convenient and better performance in position tracking control. What's more, this test rig is built by our group and is under the test in the laboratory. In later papers we will discuss about its achievements in the helicopter gearbox test. In figure 8 the built test rig is shown. 


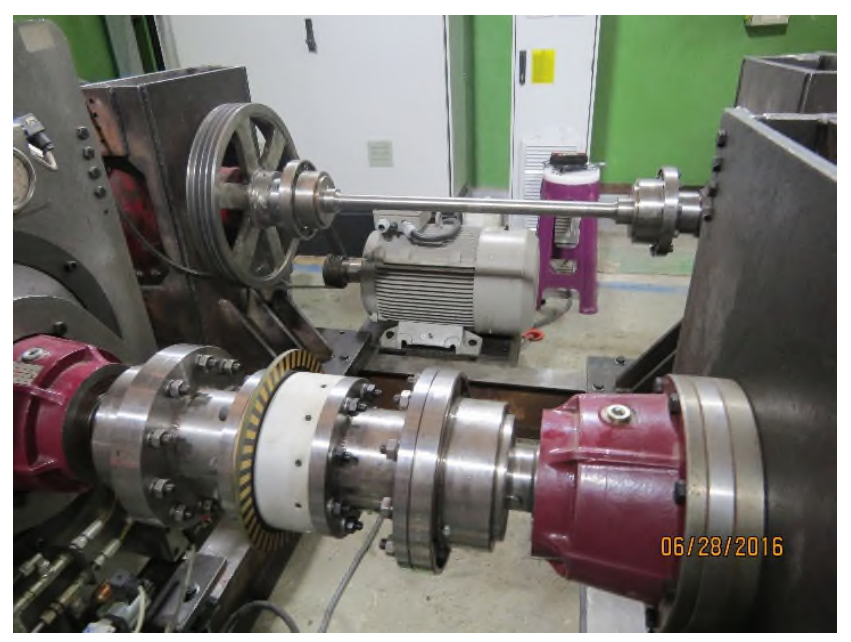

Fig. 8: Built Test Rig

\section{Conclusion}

In this paper a mechanical closed loop is used for testing a gearbox. The torque induction element was a planetary gearbox which induced torque in the close loop by changing into of its ring angle. Results show that PID controller is more accurate, and accelerated. For this purpose two kinds of hydraulic actuator are used and their model are derived and verified by the software. Moreover, the control parameter in these two methods was hydraulic jack's stroke. By this the controller could control the pressure behind the hydraulic jack's piston.

\section{References}

[1] Norlela Ishak, Mazidah Tajjudin, Hashimah Ismail, Mohd Hezri Fazalul Rahiman, Yahaya Md Sam, Ramli Adnan," PID Studies on Position Tracking Control of an Electro-Hydraulic Actuator", International Journal of Control Science and Engineering 2012, 2(5): 120-126 DOI: 10.5923/j.control.20120205.04.

[2] Mohammed Ahmed, Borskghinchin Daniel Halilu, Musa Idi and Babayo Mohammed," System Identification and Control of A Hydraulic Actuator", ARPN Journal of Engineering and Applied Sciences, VOL. 10, NO. 19, OCTOBER 2015, ISSN 1819-6608.

[3] Ayman A. Aly," Model Reference PID Control of an Electro-hydraulic Drive", I.J. Intelligent Systems and Applications, 2012, 11, 24-32 Published Online October 2012 in MECS (http://www.mecs-press.org/) DOI: 10.5815/ijisa.2012.11.03.

[4] Lingjun Li, Thomas Thurner," Accurate Modeling and Identification of Servo-Hydraulic Cylinder Systems in Multi-Axial Test Applications", Proceedings of the UKSim-AMSS Eighth European Modelling Symposium. (2014), S. 297 - 302.

[5] Toikkanen, Jari, Rummakko, Markku, "A test rig and a method for testing gearboxes and electrometrical energy converters", EP 2574778 A1.

[6] http://www.moog.com/literature/ICD/dpcmeseriesvalves.pdf

[7] W. J. Thayer, Transfer Function for MOOG Servovlves, Technical Bulletin 103, MOOG. 
[8] Kyoung Kwan AHN, Dinh Quang Truong, Yoon Hong, "Self Tuning Fuzzy PID Control for Hydraulic Load Simulator", International Conference on Control , Automation and Systems 2007 Oct. 17-20,2007 in COEX, Seoul, Korea.

[9] Asst. Lect. Munaf F. Bader, "Position Control System of Hydraulic Cylinder Based on Microcontroller", Journal of ngineering and Development, Vol. 12, No. 3, September (2008) ISSN 1813-7822.

[10] Qin Zhang," Hydraulic Linear Actuator Velocity Control Using A Feedforward-Plus-Pid Control", Int, J Flex Autom Integr Manuf 7(3):277-292.

[11] Lingjun Li, Uwe Poms, Thomas Thurner," Accurate Position Control of a Servo-Hydraulic Test Cylinder by Iterative Learning Control Technique", 2014 UKSim-AMSS 8th European Modelling Symposium.

[12] D. Xue, C. Zhao, and Y. Q. Chen, "Fractional Order PID Control of a DC Motor with Elastic Shaft: A Case Study," Proc. of the 2006 American Control Conference, Minneapolis, Minnesota,USA, June 14-16, 2006, pp. 3182-3187.

[13] A. Oustaloup, F. Levron, B. Mathieu, and F. M. Nanot, "Frequency-Band Complex Non integer Differentiator: Characterization and Synthesis," IEEE Transactions on Circuits and Systems I: Fundamental Theory and Applications, vol. 47, no. 1, 2000, pp.25-39.

[14] I. Podlubny, "Fractional-order systems and PI $\lambda D \mu$ controllers," IEEE Transactions on Automatic Control, vol. 44, no. 1, 1999, pp.208-214.

[15] B. M. Vingare, I. Podlubny, L. Dorcak, and V. Feliu, "On fractional PID controllers: A frequency domain approach," IFAC workshop on digital control. Past, present and future of PID control, 2000, pp.53-58.

[16] Y. Q. Chen, and K. L. Moore, "Discritization schemes for fractional order differentiators and integrators," IEEE Transactions on Circuits and Systems I: Fundamental Theory and Applications, vol. 49, no. 3, 2002, pp.363-367. 